ISSN: 2386-3919 - e-ISSN: 2386-3927

DOI: https://doi.org/10.14201/et20183614161

\title{
ANÁLISIS DEL MATERIAL CURRICULAR EDITADO DE EDUCACIÓN INFANTIL Y SU USO EN EL AULA
}

\author{
Analysis of the edited curricular material for early \\ chilhood education and its use in the classroom
}

\author{
Teresa Alonso Pedrosa* y Rosa M. ${ }^{a}$ SANTAMARÍA CONDE*** \\ *Maestra de Educación Infantil. CEIP Fernando de Rojas. Burgos (España) \\ Correo-e:webtere@botmail.com \\ * Profesora Titular de Universidad. Departamento Ciencias de la Educación. \\ Facultad de Educación. Universidad de Burgos (España) \\ Correo-e: rsantamaria@ubu.es
}

Recibido: 21.11.2017; Aceptado: 10.1.2018; Publicado: 30.6.2018

Ref. Bibl. TERESA ALONSO PEDROSA y ROSA M. ${ }^{a}$ SANTAMARÍA CONDE. Análisis del material curricular editado de Educación Infantil y su uso en el aula. Enseñanza E Teaching, 36, 1-2018, 41-61.

RESUMEN: La Educación Infantil, aun no siendo obligatoria en nuestro sistema educativo, constituye una etapa de gran importancia en el desarrollo del niño, y de hecho la mayor parte de la población infantil entre los tres y los seis años de edad está escolarizada. Por ello, en esta ocasión se realiza un análisis de los materiales curriculares que han sido publicados por las distintas editoriales y la implicación que ha supuesto su utilización en las aulas, constituyendo la primera aproximación del niño al proceso de enseñanza-aprendizaje a través, sobre todo, de material impreso. El estudio se ha desarrollado en el segundo ciclo de Educación Infantil, tomando como referencia los centros educativos públicos de Burgos (la mayor parte son centros que también imparten Educación Primaria). Seleccionado dicho material bibliográfico, se ha procedido a un estudio categorial analizando las siguientes variables: material para uso del alumnado y del profesorado en el aula, centros de interés, análisis del ámbito descriptivo, de la función de las intenciones educativas, de los materiales con propuestas de distintas actividades, de la atención a la diversidad y, por 
último, los aspectos formales. Los datos revelan que un alto porcentaje de los materiales curriculares son editados en forma de paquete curricular y en menor medida en material autosuficiente (proyectos). El material editado en este periodo presenta sus contenidos en forma de programación globalizada en una amplia mayoría, apareciendo los contenidos de manera superpuesta en torno a un centro de interés y no completamente globalizados. Encontramos toda una diversidad de materiales que responden a las necesidades de los docentes, si bien, podemos afirmar que no se ha encontrado material alguno que agrupe su trabajo sin diferenciar apartados, lo cual supone una posterior reorganización contextualizada de dicho material para atender a las características concretas del alumnado.

Palabras clave: educación infantil; materiales curriculares; recursos materiales; editoriales; aula.

SUMMARY: Early childhood education, although not being compulsory in our educational system, constitutes a stage of great importance in the development of the child, and of fact most of the children between three and six years old are enrolled in school. For this reason, an analysis of the curricular materials that have been published by the different publishing and the implication of their use in the classrooms has been developed, constituting the first approximation of the child to the process of the teaching-learning process through printed material. The study was developed in the second cycle of Early Childhood Education, taking as a reference the public educational centers of Burgos (most they are centers that also give Primary Education). Once the bibliographic material has been selected, a categorical study has been carried out analyzing the following variables: material for the use of the students and teachers in the classroom, centers of interest, analysis of the descriptive scope, the function of the educational intentions, the materials with proposals for different activities, attention to diversity, and finally, formal aspects. The data reveal that a high percentage of the curricular materials are edited in the form of a curricular package and to a lesser extent in self-sufficient material (projects). The material published in this period presents its contents in the form of globalized programming in a largemajority, appearing the contents in a superimposed way around a center of interest and completely globalized. We find a variety of materials that respond to the needs of teachers, although, we can confirm that no material has been found to group the work without differentiating sections, which means a subsequent contextualized reorganization of this material to meet the specific characteristics of the students.

Key words: infantile education; curricular materials; material resources; publishing houses; classroom.

\section{INTRODUCCIÓN}

El convencimiento de que muchos de los aprendizajes que vendrán a lo largo de toda nuestra vida tienen su base primordial en los años de la etapa infantil no es sino un motor muy fuerte para poder seguir estudiando acerca de los mismos. 
La mayor parte de las habilidades que utilizamos en un día se basan en aprendizajes adquiridos en los primeros años de nuestra vida. Por ello, el objetivo de la etapa educativa no puede ser otro que el de acompañar al alumnado en todos sus aprendizajes para conseguir un desarrollo global e íntegro de su persona.

Gútiez (1995: 102) señala que la infancia tiene una gran importancia en el proceso de maduración del individuo, ya que en los tres primeros años de vida se va a desarrollar la mitad del potencial intelectual del ser humano, es decir, que la capacidad y necesidad de desarrollo intelectual es mayor cuanto menor sea la edad del niño y, consecuentemente, las posibilidades de recibir una atención adecuada van a determinar su desarrollo futuro. Un fracaso en estas etapas supone un factor determinante en la aparición del fracaso escolar. Por su parte, Jaramillo (2007: 110) entiende por primera infancia el periodo de la vida de crecimiento y desarrollo comprendido desde la gestación hasta los siete años aproximadamente y que se caracteriza por la rapidez de los cambios que ocurren. Esta primera etapa es decisiva en el desarrollo, pues de ella va a depender toda la evolución posterior del niño en las dimensiones motora, lenguaje, cognitiva y socioafectiva, entre otras.

Desde que diversos autores comenzaron a plantearse la importancia de la educación en las primeras etapas, surgen como tales diversas corrientes que plantean la necesidad de crear el estudio de esta etapa de la vida y generar una serie de criterios para establecer una etapa con entidad, objetivos, principios y cuerpo propios. Surgen, a través de diversas leyes y órdenes distintas pinceladas, que le van dando identidad propia. Es así como se establece como tal una etapa en la que el desarrollo del niño de 0 a 6 años es el centro. En la Ley de Ordenación General del Sistema Educativo Español (LOGSE, 1990), se llevó a cabo un avance significativo al considerar esta etapa como el primer tramo en el sistema educativo, aspecto que se ha corroborado posteriormente con la Ley Orgánica de Educación (LOE, 2006) y la Ley Orgánica para la Mejora de la Calidad Educativa (LOMCE, 2013).

Durante todos estos años, nos damos cuenta de que los principios que rigen la educación infantil no son estancos, tienen que fluir y cambiar de acuerdo con la evolución de los tiempos en los que los alumnos viven, crecen y se educan. Gracias a los diferentes estudios médicos, sociológicos, psicológicos y pedagógicos, somos conscientes de la importancia de los rapidísimos cambios fisiológicos, del gran desarrollo de la etapa afectiva, del trepidante desarrollo motor, de los múltiples cambios afectivos y sociales que se desarrolla en torno al niño (apego, egocentrismo, apoyo entre iguales, dependencia del adulto, conquista de la autonomía...) es una etapa en la que se marcan las primeras pautas de actuación y en la que se marca el desarrollo integral posterior del alumno.

Si nos centramos más concretamente en el segundo ciclo de Educación Infantil, en las edades de 3 a 6 años, nos encontramos con alumnos que han tenido tres años de experiencias familiares y sociales muy diversas, en las que unos meses de vida en ocasiones suponen grandes diferencias en el desarrollo; alumnos que han o no tenido una experiencia en las escuelas infantiles y/o en guarderías, una etapa no obligatoria, pero cada vez considerada más importante por los profesionales e 
imprescindible por las familias, tanto a nivel educativo como necesario para conciliar de la manera más adecuada posible su vida laboral y familiar. En conclusión, «es en la primera infancia donde las oportunidades sociales, económicas y culturales nos igualan o diferencian" (Castañeda, 2010: 32). Así pues, se debe considerar la Educación Infantil como la etapa idónea para desarrollar hábitos que ayuden a conseguir un equilibrio emocional en los infantes (Martínez, Pérez y Sierra, 2014: 152).

Argos, Ezquerra y Castro (2011: 1) afirman que, en los últimos años, se ha producido un incremento en el interés por escuchar la voz del niño y su perspectiva dentro del ámbito educativo. Se entiende esta participación activa como fruto de la nueva concepción existente sobre la infancia. Este nuevo concepto de infancia emergente presenta al niño como un actor social de pleno derecho, en vez de un sujeto pasivo dentro de la sociedad.

Diversos y numerosos trabajos son los que han asumido cómo enfrentarse a esta etapa educativa y a los materiales curriculares que se utilizan en el devenir diario de dichas aulas. Vamos a tratar de esclarecer cómo son los materiales que llegan a las manos de los niños del segundo ciclo de Educación Infantil y cuál es el uso que se hace de ellos en las aulas.

\section{LA EDUCACiÓN INFANTIL A tRAVÉS DE lOS MATERIALES CURRICUlares DE LAS EDITORIALES}

Primeramente, antes de recoger cómo los materiales entienden la etapa de Educación Infantil, y ante la siempre controvertida elección entre si usar o no materiales curriculares por nuestra parte, tras valorar muchos aspectos positivos y negativos que aportan múltiples estudiosos al respecto, recogemos una reflexión de Martínez (2001: 24), con la que estamos totalmente de acuerdo, ya que son muchos los movimientos que se han generado en los últimos años en torno a la gratuidad de los libros y hacia la no desaparición de los mismos, debido al prestigio que les otorgan las familias y las editoriales, mas son mínimos los esfuerzos que se dedican a valorar su cientificidad, su validez o su calidad. Ante el convencimiento de que no nos podemos convertir en consumidores pasivos e interesados desconocedores de los mismos, tratamos de aportar con este trabajo un granito más a los escasos estudios realizados a este respecto. Así, Martínez (2001: 24) se plantea que, en nuestro país, el interés por una teoría general del libro de texto es prácticamente nulo, si lo juzgamos a la luz de la producción científica publicada al respecto.

Esta situación se agrava aún más en la etapa de Educación Infantil, en la que el material curricular es considerado fungible y, por tanto, de usar y tirar, con todos los aspectos positivos y negativos que ello implica. Si bien, los estudios sobre su cientificidad o valor para el uso por parte del alumnado quedan completamente en el olvido.

Es el propio profesorado de Educación Infantil, en un cada vez más denostado tiempo para programar, el que tiene que decidir la adecuación de los materiales a las circunstancias sociales, económicas o particulares del alumnado de su centro. Eso sí, cada vez antes, para facilitar el trabajo a las familias y editoriales y quizás 
para niños que no serán tus alumnos en el curso próximo. En resumen, se usen o no, se utilicen como herramienta o como pilar de nuestras enseñanzas, como ayuda o como impedimento para los aprendizajes, lo más valioso es que los materiales estén dotados de una cientificidad adecuada y adaptada a la edad del alumno que lo recibe, ya que será el primer referente textual de su escolaridad. Además de pegatinas o colores, o elementos atractivos variados, lo más interesante para el profesional que lo reciba es que estén elaborados con un rigor científico adaptado a los alumnos que los van a utilizar.

Así, Salgado y Salinas (2009: 488) afirman que los libros de texto están presentes en muchas aulas de Educación Infantil y aseguran que su uso hace que jueguen un papel importante en la construcción de conocimientos. Recogen que los maestros de Educación Infantil, según Martínez (1992), son los que a partir de su práctica educativa determinan la mayor parte de los procesos de aprendizaje y en muchas ocasiones los procedimientos que se siguen son los que exponen los libros de texto. La preocupación didáctica de los libros de texto fue hasta hace pocos años técnica, se centraba en aspectos formales y estructurales como son entre otros diseño, formato, tipo de letra e ilustraciones.

\section{DisEÑO DE LA INVESTIGACiÓN}

A continuación, presentamos el diseño de la investigación centrada en el estudio de los materiales curriculares del segundo ciclo de Educación Infantil de las principales editoriales.

\subsection{Objetivos}

La finalidad que pretendemos con este estudio es conocer la mayor parte de los materiales curriculares editados en torno al cambio legislativo del año 2006, Ley Orgánica de Educación (LOE, 2006), y aquellos que editados en años anteriores han sido utilizados en los centros de la provincia de Burgos durante el periodo de su desarrollo, así como los editados posteriormente en el periodo post-LOE mediante una descripción y análisis sistemático del contenido y forma de dichos materiales.

Para ello, nos basaremos en el método de investigación aportado por Bardin (1986), que incluye un conjunto de técnicas para el análisis de las comunicaciones. El análisis de contenido, como técnica de investigación, reúne los requisitos metodológicos para investigar la naturaleza del discurso, analizar y cuantificar los materiales que intervienen en los procesos de comunicación. Nuestro propósito es obtener un conocimiento objetivo de cada uno de los materiales analizados y realizar una valoración de los mismos a través del análisis de contenido, como técnica de investigación.

Además, tal como afirman Delgado y Rodríguez (2011: 109) recogiendo las palabras de Castiello (2002: 207), el análisis de contenido puede estar dirigido a la 
"descripción o a la formulación de inferencias». Según el mismo autor, el análisis inferencial «tiene por objeto analizar el contenido de una comunicación con el fin de formular y describir cuestiones y reglas que, guardando una relación con el contenido, van más allá de su formulación explícita, adquiriendo entonces la investigación un carácter explicativo; por lo que a través del análisis de los resultados conclusiones trataremos de establecerlas.

Para dar solución a estos y otros interrogantes nos hemos planteado los siguientes objetivos:

- Conocer los materiales curriculares que se utilizan en el segundo ciclo de Educación Infantil de los centros públicos de Burgos.

- Analizar en profundidad estos materiales, en concreto, los presentados en soporte papel, y sus materiales complementarios, desde la puesta en vigor de la Ley Orgánica 2/2006, de 3 de mayo, de Educación (LOE, 2006).

Para profundizar en el análisis, nos planteamos:

- Conocer el material del profesorado, del aula y del alumnado.

- Destacar los centros de interés más utilizados.

- Analizar el ámbito descriptivo de los materiales curriculares editados.

- Valorar las intenciones educativas.

- Enfatizar cómo son los materiales con propuestas de aprendizaje.

- Valorar la atención a la diversidad del alumnado.

- Analizar los aspectos formales.

Dichos objetivos se llevarán a cabo, principalmente, a través de las tareas de recoger y analizar cuál es la situación general de la enseñanza en la etapa de Educación Infantil y la reflexión sobre la situación actual de la enseñanza en la etapa de Educación Infantil.

\subsection{Población y muestra}

En este apartado presentamos, de forma objetiva, minuciosa y descriptiva el contenido y los aspectos más relevantes de los libros de texto utilizados en las aulas de segundo ciclo de Educación Infantil de los centros de Burgos. Esta descripción pretende lograr una perspectiva aproximada a los textos, con objeto de resaltar individualmente sus aspectos más identificativos. Con este estudio se pretende obtener conocimientos válidos para seleccionar los materiales que van a ser objeto de un análisis más pormenorizado y así poder extraer las categorías y subcategorías que serán analizadas en el siguiente apartado, estando claros varios criterios de preselección, que hicieran delimitar el corpus o conjunto de materiales objeto de análisis, y que a continuación se detallan:

Materiales curriculares utilizados en las aulas entre los años 2006-2015. Por ello, su edición está en torno a dichos años, si bien, hemos añadido algunos textos 
que, aunque están editados en años anteriores, han sido utilizados en las aulas en dicho periodo.

Se delimita la muestra en el segundo ciclo de Educación Infantil, ya que, actualmente, en los centros de Educación Infantil y Primaria solo se ha incorporado este ciclo educativo. Aunque si bien es cierto que cada vez más en el primer ciclo de Educación Infantil se utilizan los materiales impresos, la mayor parte surge de paquetes metodológicos que ya se usaban en el segundo ciclo, y se elaboran de manera similar. Por lo que en este estudio nos centraremos solo en el material de este segundo ciclo.

Solo se han seleccionado los textos escritos en castellano y editados para ser usados en Castilla y León, ya que es el ámbito territorial y lingüístico de nuestra ciudad de Burgos. Los materiales que vamos a seleccionar para nuestra muestra son materiales impresos y que traten los aprendizajes de manera globalizada. De este modo, los volúmenes que son objeto de nuestra investigación aparecen reflejados en la siguiente tabla:

TABLA 1

Títulos de los distintos proyectos y su correspondiente editorial que son objeto de estudio (elaboración propia)

\begin{tabular}{|l|c|}
\hline \multicolumn{1}{|c|}{ TíTULO DEL PROYECTO } & EDITORIAL \\
\hline ¿Qué idea! / Cachalote / Proyectos / Monigotes & ANAYA \\
\hline Juntos con Lola, Max y Zeta / Otito, Ota y yo / Lobo Rojo & BRUÑO \\
\hline Torbellinos / Bichitos / Todos al agua & CASALS \\
\hline Kids / Proyecto Cucú / TicTac & EDEBÉ \\
\hline $\begin{array}{l}\text { Proyecto Nubaris / Rumbo Nubaris / Dimensión Nubaris / Tocalotodo } \\
\text { / ¿Lo ves? }\end{array}$ & EDELVIVES \\
\hline Papelillos / Papapapú / Duendes Mágicos / Proyectos / Pompas de jabón & ALGAIDA \\
\hline $\begin{array}{l}\text { En ruta con Peky / Peky explora / Cuentoaventura / Daniel y los } \\
\text { Diversónicos / Peky / Fantasía / Luna Llena / Nuevo Magos y Genios / } \\
\text { Mundo Flopi / Pro + }\end{array}$ & EVEREST \\
\hline Alethea. La isla de Cucas, Eli y Pan / Exploradores / Meñiques & OXFORD \\
\hline $\begin{array}{l}\text { El viaje de Suso / Mica y sus amigos / Nuevo Cocorolos / Birli - Birloque } \\
\text { / Chiribitas / Cuánto Sabemos }\end{array}$ & SANTILLANA \\
\hline $\begin{array}{l}\text { Guau / Colorines / Volteretas / A la de tres / Volteretas y más vueltas / } \\
\text { El cole viajero }\end{array}$ & S.M. \\
\hline Espiral Mágica & VICENS VIVES \\
\hline
\end{tabular}

En total, se han seleccionado un total de 49 materiales y/o libros de texto, de 11 editoriales, que han sido publicados y/o utilizados en los centros de Burgos, durante los cursos 2006-2015. 
Posteriormente, como establecía Krippendorff (1990), pasaremos a su descomposición, que supone fijar cuáles van a ser las unidades que vamos a tener en cuenta y qué elementos vamos a observar. A continuación, nos centramos en las normas de enumeración o forma de identificar las categorías. En nuestro análisis categorial, hemos tenido principalmente en cuenta la presencia o ausencia de la categoría (sí, no o en blanco cuando se desconoce) y la frecuencia ponderada de las mismas. Con respecto a la categorización, hemos establecido nueve categorizaciones con diversas subcategorías cada una. Para ello, utilizaremos un modelo propuesto por Parcerisa (1996). A través de esta herramienta conseguiremos operativizar el análisis de todos los materiales que se utilizan en las aulas y que han aparecido en el mercado desde la puesta en marcha de la Ley Orgánica de Educación (LOE, 2006), dirigidos al segundo ciclo de este nivel educativo.

\subsection{Instrumento}

Se usa el sistema categorial para el análisis de los libros de texto y el material curricular, tratando de describir la fiabilidad y validez del sistema categorial, para posteriormente dar paso al estudio categorial en sí de la muestra elegida de los materiales curriculares en el periodo de 2006 a 2015. Para ello se han determinado varias tablas de registro en las que se han detallado las frecuencias de la aparición de las distintas categorías delimitadas para su estudio. Elaboradas las hojas de registro definitivas, partiendo, como se ha comentado, de las aportadas por Parcerisa (1996), se aplicaron a los materiales de texto recogidos en la investigación. Este registro se ha realizado de manera sistemática, siguiendo las orientaciones emanadas de la aplicación del análisis categorial. Finalmente, se ha realizado un recuento y cómputo de las frecuencias relacionadas con cada una de las categorías. Los resultados obtenidos se presentan porcentualmente y se valoran, a tenor de las categorías presentadas.

\subsection{Procedimiento de recogida y análisis de datos}

Siguiendo el enfoque del análisis de contenido, hemos tenido en cuenta las siguientes fases:

- Planteamiento de interrogantes y objetivos de la investigación.

- Justificación de la muestra: material utilizado en el periodo 2006-2015.

- Metodología.

- Conclusiones.

El análisis de contenido es una de las técnicas para el análisis de comunicación humana utilizadas tradicionalmente para decodificar los mensajes manifiestos, latentes y ocultos plasmados en diferentes documentos. Como técnica asume como principio que los documentos reflejan las actitudes y creencias de las personas e 
instituciones que los producen, así como las actitudes y creencias de los receptores de estos (Cabero y Loscertales, 2002: 1). Utilizaremos la adecuación de las fases, propuestas por Bardin (1986), siguiendo los pasos establecidos por Boronat (2005: 171). En su trabajo sobre la aplicación del análisis de contenido a los suplementos educativos de la prensa, que contempla las siguientes fases, que hemos aplicado en nuestro estudio:

- Delimitación de los objetivos de la investigación.

- Elección de la muestra: selección de los documentos.

- Determinación de las unidades de análisis.

- Delimitación de las categorías: elaboración de una guía.

- Análisis e interpretación de los datos.

- Elaboración de los resultados y conclusiones.

\section{Resultados}

A continuación, mostramos los resultados de manera porcentual, determinándolos de forma individual, para, posteriormente, realizar conclusiones más generales de cada apartado que nos ayuden a la interpretación e interrelación de los datos obtenidos del estudio de la muestra. Para poder revisar y analizar de manera adecuada y objetiva los datos obtenidos de los cuadros categoriales que hemos diseñado, hemos ordenado cada uno de los aspectos valorados a través de las frecuencias de manera descendente.

TABLA 2

Porcentaje del material para uso del alumnado

\begin{tabular}{|c|l|}
\hline $100 \%$ & Cuadernos de actividades. \\
\hline $89.79 \%$ & Carpeta o archivador. \\
\hline $89.8 \%$ & Adhesivos o gomets. \\
\hline $79.60 \%$ & $\begin{array}{l}\text { Información para la familia: Informe para la familia, revista o carta para la familia, } \\
\text { pasaporte de puntos, cuestionario inicial. }\end{array}$ \\
\hline $77.55 \%$ & Láminas de plástica y/o láminas de fiesta. \\
\hline $75.51 \%$ & Cuentos o libros de lectura. \\
\hline $61.22 \%$ & $\begin{array}{l}\text { Material de matemáticas, lectoescritura, ciencia y/o desarrollo de capacidades } \\
\text { y/o emociones. }\end{array}$ \\
\hline $44.9 \%$ & Troqueles y/o recortables. \\
\hline $42.86 \%$ & CD o DvD. \\
\hline $38.78 \%$ & Grafomotricidad. \\
\hline $18.37 \%$ & Caretas, llaveros, peluches, mascotas, etc. \\
\hline $12.24 \%$ & Murales. \\
\hline
\end{tabular}


En cuanto al material dedicado a ser usado por el alumnado, podemos observar que solo hay un aspecto que es general al 100\% de los materiales, y es el cuaderno de actividades, si bien, como iremos analizando los cuadernos difieren mucho en sus características entre sí. Como hemos podido valorar, primeramente la mayor parte de los materiales tienen tres cuadernos por trimestre (29), seguido de los que disponen de dos cuadernos por trimestre (10), en menor número los materiales que utilizan un cuaderno por cada proyecto (7) y, finalmente, aquellos que disponen de unidades didácticas de manera más variada (3).

TABLA 3

Porcentaje del material para uso del profesorado (elaboración propia)

\begin{tabular}{|c|l|}
\hline $100 \%$ & Propuesta didáctica. Libro de aula o guía. \\
\hline $83.68 \%$ & Material de apoyo didáctico. \\
\hline $79.60 \%$ & CD de recursos. \\
\hline $42.86 \%$ & Cuaderno de evaluación. \\
\hline $38.78 \%$ & Propuesta de psicomotricidad. \\
\hline $38.78 \%$ & Propuesta de recursos orales. \\
\hline $32.65 \%$ & Propuesta de plástica. \\
\hline $26.53 \%$ & $\begin{array}{l}\text { Propuestas: de números, ciencias, valores, talleres rincones, proyecto o } \\
\text { grafomotricidad. }\end{array}$ \\
\hline $22.45 \%$ & Propuesta de música. \\
\hline $4.08 \%$ & Material del mundo. \\
\hline
\end{tabular}

En el 83.68\% de los materiales encontramos material de apoyo didáctico, que bien puede estar referido a las actividades de refuerzo y/o de ampliación. No obstante, aunque en muchos casos aparece de manera complementaria en cuadernos anexos o en un su apartado dentro de la propia guía didáctica o libro de aula, en la mayor parte de los casos están centradas solo en el desarrollo de actividades o fichas fotocopiables. En un $79.60 \%$ los materiales referidos a su uso para el profesorado disponen de CD de recursos; en ellos, encontramos, en orden de mayor a menor: recursos, programación, actividades para la pizarra digital interactiva, proyecto curricular y materiales on-line. 
TABLA 4

Porcentaje del material para uso en el aula (elaboración propia)

\begin{tabular}{|l|l|}
\hline $91.84 \%$ & Láminas murales, calendario, cumpleaños y otros. \\
\hline $73.47 \%$ & Mascota. \\
\hline $69.39 \%$ & CD o DVD interactivo o libro digital. \\
\hline $65.30 \%$ & Cuento gigante. \\
\hline $65.31 \%$ & Bits, tarjetas de valores, de vocabulario, arte, hábitos, etc. \\
\hline $61.22 \%$ & Juegos y habilidades manipulativas. \\
\hline $55.10 \%$ & Caja contenedor. \\
\hline $34.69 \%$ & Letras troqueladas, abecedario, números, formas, etc. \\
\hline $18.37 \%$ & Premios o registros de conducta. \\
\hline $10.20 \%$ & Póster organizador de aula. \\
\hline
\end{tabular}

Como podemos apreciar en alguno de los puntos, hay aspectos que a la hora de categorizarlos nos han sido de mayor dificultad, ya que muchas veces aparecen mezclados como material de profesor y/o del aula. A continuación, exponemos los resultados obtenidos atendiendo a los siguientes aspectos: centros de interés, ámbito descriptivo (intenciones y ámbito de aplicación), ámbito de análisis en función de las intenciones educativas, ámbito de análisis en función de los requisitos para el aprendizaje, ámbito de análisis en función a la atención a la diversidad y, por último, ámbito de análisis en función de los aspectos formales. Si ordenamos de manera descendente el uso que hacen los materiales curriculares estudiados, de las temáticas, según las veces que son usados como centro de interés en sus unidades didácticas o proyectos de trabajo, encontramos: 
TABLA 5

Centros de interés por porcentajes (elaboración propia)

\begin{tabular}{|l|l|l|}
\hline 93.88\% Animales & $38.78 \%$ Viajes & $16.33 \%$ El mercado \\
\hline $77.55 \%$ Las plantas & $38.78 \%$ La primavera & $14.29 \%$ Fenóm. atmosféricos \\
\hline $75.51 \%$ El colegio & $38.78 \%$ El paisaje & $\begin{array}{l}14.29 \% \text { Niños y niñas del } \\
\text { mundo }\end{array}$ \\
\hline 69.39\% La casa & $36.73 \%$ Ocio y tiempo libre & $\begin{array}{l}14.29 \% \text { Los continentes y } \\
\text { océanos }\end{array}$ \\
\hline 67.35\% La calle & $36.73 \%$ El invierno & $12.24 \%$ El reciclaje \\
\hline 67.35\% El cuerpo & $\begin{array}{l}34.70 \% \text { Higiene y cuidado } \\
\text { del cuerpo }\end{array}$ & $12.24 \%$ La ropa \\
\hline $59.18 \%$ Los transportes & $32.65 \%$ Historia & $12.24 \%$ Dinosaurios \\
\hline $\begin{array}{l}55.10 \% \text { Los medios de } \\
\text { comunicación }\end{array}$ & $30.61 \%$ Los oficios & $8.16 \%$ Las emociones \\
\hline $55.10 \%$ El verano/vacaciones & $28.58 \%$ La granja & $8.16 \%$ El coche \\
\hline $53.06 \%$ Juegos y disfraces & $\begin{array}{l}28.57 \% \text { Arte y los artistas. El } \\
\text { museo }\end{array}$ & $6.12 \%$ Música \\
\hline $53.06 \%$ Los alimentos & $24.49 \%$ Biblioteca y libros & $6.12 \%$ Elementos de la vida \\
\hline $51.02 \%$ La Navidad & $20.41 \%$ Deportes & $6.12 \%$ El teatro \\
\hline $48.98 \%$ El sistema solar & $20.40 \%$ Los sentidos & $2.04 \%$ El reloj-el tiempo \\
\hline $42.86 \%$ La familia & $18.37 \%$ Los inventos & $2.04 \%$ Las elecciones \\
\hline $40.81 \%$ El otoño & $16.33 \%$ Carnaval & \\
\hline
\end{tabular}

Aclarar que ciertas unidades didácticas o proyectos pueden en algún momento referirse a alguna de estas temáticas como contenido, pero no como centro de interés, por lo que no han sido recontadas en este punto. Nos ha llamado extraordinariamente la atención la gran dispersión de centros de interés que se trabajan en los materiales curriculares, habiendo solamente doce de las cuarenta y cuatro encontradas que tengan una frecuencia de uso superior al 50\%. Hemos podido observar, también, que las temáticas utilizadas por las editoriales como centros de interés, en su mayor parte, siguen siendo las tradicionalmente usadas, si bien, se actualizan sus contenidos. 
TERESA ALONSO PEDROSA Y ROSA M. ${ }^{\text {a }}$ SANTAMARÍA CONDE

TABLA 6

Porcentajes derivados del ámbito descriptivo (elaboración propia)

\begin{tabular}{|c|l|l|l|}
\hline $12.24 \%$ & Es autosuficiente. & $87.76 \%$ & Paquete curricular. \\
\hline $87.76 \%$ & Desarrollan objetivos y contenidos de un ciclo. & $12.24 \%$ & De un curso. \\
\hline $100 \%$ & Desarrolla objetivos y contenidos de varias áreas. \\
\hline $100 \%$ & Está constituido por varios componentes. \\
\hline $100 \%$ & Es fungible. \\
\hline $100 \%$ & De uso individual. \\
\hline $100 \%$ & Dividido en unidades o temas. \\
\hline $100 \%$ & La información para realizar las actividades se encuentra en el propio texto. \\
\hline $20.51 \%$ & Se propone recurrir a otros materiales. \\
\hline $86.49 \%$ & Recurrir a otros materiales siendo sugeridos en el paquete curricular. \\
\hline $94.87 \%$ & Sugieren posibilidades de ampliación. \\
\hline $95.92 \%$ & Tratan de correlacionarlos (superponen). \\
\hline $97.37 \%$ & Programación globalizada. (Poco profunda y escasa. A través de un nexo). \\
\hline
\end{tabular}

TABLA 7

Porcentajes del ámbito de análisis en función de las intenciones educativas (elaboración propia)

\begin{tabular}{|c|l|}
\hline $97.22 \%$ & Los contenidos se encuentran actualizados. \\
\hline $94.44 \%$ & Objetivos didácticos son adecuados a la edad del alumno. \\
\hline $73.67 \%$ & Las ilustraciones son coherentes con los ejes transversales. \\
\hline $72.22 \%$ & Se plantean objetivos y/o contenidos correspondientes a ejes transversales. \\
\hline $68.42 \%$ & $\begin{array}{l}\text { El tono, las afirmaciones y los ejemplos del texto son coherentes con los valores } \\
\text { asumidos en el de los materiales. }\end{array}$ \\
\hline $42.86 \%$ & Opciones tomadas en el material estén explícitamente justificadas. \\
\hline $25.71 \%$ & $\begin{array}{l}\text { Los contenidos aparecen diferenciados. (conceptual, procedimental y actitudinal- } \\
\text { LOGSE 1990). }\end{array}$ \\
\hline $16.67 \%$ & Contenidos correlacionados entre sí. \\
\hline $100 \%$ & Los contenidos se corresponden con los objetivos. \\
\hline $100 \%$ & Actividades que se corresponden con los objetivos y contenidos. \\
\hline $80 \%$ & Las propuestas de evaluación se encuentran en función de los objetivos. \\
\hline $50 \%$ & Actividades para facilitar el aprendizaje de los ejes transversales. \\
\hline
\end{tabular}

En cuanto al ámbito de análisis en función de los requisitos para el aprendizaje, nos cabe destacar, inicialmente, que son solo 14 los métodos que adjuntan para el alumnado materiales informativos (dichos materiales difieren mucho en la 
forma y modo de abordar la información, más si se trata de pequeños libros-enciclopedias, que intentan aumentar la lectura y el aprendizaje de tipo científico de los alumnos). Dentro de los que hemos podido encontrar, diremos que el 92.86\% dispone de un nivel lingüístico adecuado a la edad del alumno y el $85.71 \%$ dispone de una densidad informativa adecuada.

TABLA 8

Porcentajes de los materiales con propuestas de actividades (elaboración propia)

\begin{tabular}{|c|l|}
\hline $97.56 \%$ & Actividades de búsqueda de información. \\
\hline $92.68 \%$ & De contextualización y aplicación. \\
\hline $92.3 \%$ & Actividades grupales. \\
\hline $90.24 \%$ & Actividades de memorización. \\
\hline $82.86 \%$ & Criterios para la organización del aula. \\
\hline $72.5 \%$ & Evaluación inicial. \\
\hline $67.5 \%$ & Actividades de evaluación formativa. \\
\hline $47.37 \%$ & Propongan la evaluación del profesorado y del currículo. \\
\hline $43.34 \%$ & Actividades de síntesis. \\
\hline $100 \%$ & De construcción de significados. \\
\hline $100 \%$ & Actividades individuales. \\
\hline $100 \%$ & Actividades en que se plantean interrogantes que creen conflictos cognitivos. \\
\hline $100 \%$ & Actividades de motivación. \\
\hline $75 \%$ & De evaluación sumativa. \\
\hline
\end{tabular}

Los materiales de lectura aparecen de manera muy común, siendo el 93.55\% de fácil acceso para el alumnado. Prácticamente la totalidad de los materiales estudiados dispone de la estructura y longitud adecuada, así como un léxico adecuado. El 100\% vienen acompañados por una propuesta de actividades a realizar antes, durante y después de la lectura, si bien, difieren unos de otros en la forma de llevarlo a cabo.

TABLA 9

Porcentajes del ámbito de análisis en función a la atención a la diversidad (elaboración propia)

\begin{tabular}{|c|l|}
\hline $97.3 \%$ & Recoge actividades de ampliación. \\
\hline $89.19 \%$ & De refuerzo. \\
\hline $81.08 \%$ & Proponen distintas posibilidades de actividades. \\
\hline $75.68 \%$ & En las propuestas de evaluación el referente son las capacidades. \\
\hline $25.64 \%$ & Explicitan distintos niveles para la realización de las actividades. \\
\hline
\end{tabular}


TABLA 10

Porcentajes del ámbito de análisis en función de los aspectos formales (elaboración propia)

\begin{tabular}{|c|c|}
\hline $76.19 \%$ & El profesorado considera el material visualmente atractivo para el alumnado. \\
\hline $\begin{array}{c}95.12 \% \\
4.88 \% \\
\end{array}$ & $\begin{array}{l}\text { Formato en posición horizontal. } \\
\text { Formato en vertical. }\end{array}$ \\
\hline $\begin{array}{l}63.41 \% \\
75.61 \% \\
39.02 \%\end{array}$ & $\begin{array}{l}\text { Tipo de encuadernación (algunos conjugan } \\
\text { distintos tipos de encuadernación): } \\
\text { Troquelado. } \\
\text { Con agujeros. } \\
\text { Espiral. } \\
\end{array}$ \\
\hline $\begin{array}{l}26.19 \% \\
4.76 \% \\
69.05 \%\end{array}$ & $\begin{array}{l}\text { Uso del papel: } \\
\text { Utilizan solo una cara. } \\
\text { Dos caras. } \\
\text { Utilizan una o dos caras. } \\
\end{array}$ \\
\hline $28.57 \%$ & Tapas duras. \\
\hline $95.24 \%$ & $\begin{array}{l}\text { Aparece en la cubierta el título del proyecto de trabajo o de la unidad } \\
\text { didáctica y la editorial (el autor y/o el ilustrador suele aparecer en } \\
\text { la contraportada). }\end{array}$ \\
\hline $48.78 \%$ & Establece un diagrama para la distribución de los contenidos. \\
\hline $\begin{array}{l}90.48 \% \\
81.08 \%\end{array}$ & $\begin{array}{l}\text { Introduce la letra mayúscula. } \\
\text { Predomina la letra minúscula. }\end{array}$ \\
\hline $92.86 \%$ & El tamaño de letra es el ajustado. \\
\hline $100 \%$ & Utiliza un papel adecuado. \\
\hline $100 \%$ & Buen contraste entre el texto y el papel que facilita la legibilidad. \\
\hline $100 \%$ & El tipo de impresión es apropiado. \\
\hline $\begin{array}{l}90.48 \% \\
100 \% \\
97.62 \% \\
0 \% \\
\end{array}$ & \begin{tabular}{|l} 
Tipo de ilustraciones: \\
Contiene dibujos en blanco y negro. \\
Contiene dibujos en color. \\
Contiene fotografías en color. \\
Sin ilustraciones o todas son en blanco y negro.
\end{tabular} \\
\hline $46.34 \%$ & Dispone de índice. \\
\hline $0 \%$ & Aporta bibliografía. \\
\hline \multicolumn{2}{|c|}{$\begin{array}{l}\text { Precio aproximado: } \\
\text { Paquetes curriculares por trimestre de } 28 € \text {. } \\
\text { Materiales autosuficientes de } 15 € \text {. }\end{array}$} \\
\hline
\end{tabular}

Los datos reflejados en las distintas tablas son solo el reflejo de una pequeña parte que ha supuesto la investigación en su conjunto. 
TERESA ALONSO PEDROSA Y ROSA M. ${ }^{\text {a }}$ SANTAMARÍA CONDE

ANÁLISIS DEL MATERIAL CURRICULAR EDITADO DE EDUCACIÓN INFANTIL Y SU USO EN EL AULA

\section{DISCUSIÓN Y CONCLUSIONES}

Inicialmente, y partiendo de la reflexión de Ballesta (1996) que afirma que, cuando programamos las actividades de enseñanza-aprendizaje que se han de llevar a cabo en el aula, debemos tener en cuenta qué material será el más adecuado para transmitir los contenidos y alcanzar los objetivos que pretendemos y que, además, ha de ser adecuado al alumnado (por su etapa evolutiva y su estructura cognitiva) y ha de tener un valor educativo (contenido). El material que se vaya a emplear debe permitir atender aspectos importantes como: la concentración, el desarrollo de explicaciones, la participación del alumnado, el gusto estético, la posibilidad de investigación y creatividad, el desarrollo y ampliación de conocimientos, etc. Asimismo, tal como afirma Ballesta (1996), estamos de acuerdo con que una de las conclusiones del análisis de los recursos didácticos y su utilización es la necesidad de la existencia de materiales diversos y diversificables que permitan que cada profesor pueda elaborar su específico proyecto de intervención, adaptado a las necesidades de su realidad educativa y a su talante profesional. Cuanto más variados sean los materiales, más fácil será la elaboración de propuestas singulares. Por lo tanto, los proyectos de materiales curriculares para el alumnado, más que proponer unidades didácticas cerradas, tienen que ofrecer una gran variedad de recursos que puedan integrarse en unidades construidas por el propio profesorado, atendiendo las demandas de su contexto educativo.

En cuanto a la evaluación del propio profesorado y del currículo, encontramos que un $47.37 \%$ de los materiales disponen de herramientas para llevarla a cabo, mas consideramos que es un momento clave del proceso de feedback, que no debería de faltar nunca en una buena labor docente, además, no hemos encontrado ninguna herramienta que nos facilite la evaluación del propio material.

Los materiales curriculares son editados en forma de paquete curricular en un $87.76 \%$ y en material autosuficiente (proyectos) en un $12.24 \%$. El material editado en este periodo presenta sus contenidos en forma de programación globalizada en un $97.37 \%$, apareciendo los contenidos de manera superpuesta en torno a un centro de interés y no completamente globalizados; además, el 95.92\% de los materiales relacionan todos sus componentes, si bien, como ocurre con sus contenidos, aparecen muchas veces superpuestos y no completamente correlacionados. Encontramos toda diversidad de materiales que respondan las necesidades de los profesionales, si bien, podemos afirmar que no hemos encontrado ningún material que agrupe su trabajo sin diferenciar apartados, por lo cual, los profesionales que realizan su trabajo de esta manera se ven obligados a realizarlo ellos de manera personal.

Antón y Gómez (2016: 94) afirman que la condición significativa y globalizada del aprendizaje en la Educación Infantil exige contextualizarlo en un ambiente adecuado. Por ello, es necesario crear situaciones de aula que contengan los contenidos curriculares que se quiere enseñar y resulten propicias a la manipulación y experimentación por el alumnado, les resulten motivadoras y se adapten a los ritmos de pensamiento del niño. 
Con respecto a los centros de interés, hemos podido observar que se siguen proyectando los más tradicionales, de manera más frecuente por parte de las editoriales, si bien, están actualizados en un 97.22\%, lo cual facilita el trabajo a los maestros. Aunque vemos la inconveniencia de que al tratarse de materiales que se muestran cerrados, es más complicada por parte de las editoriales realizar las actualizaciones una vez plasmada la tirada.

En cuanto a los materiales curriculares, podemos afirmar que todos los materiales analizados disponen de cuaderno de actividades, para el desarrollo de fichas de carácter individual, por parte de los alumnos. Respecto al proceso de lectoescritura, los materiales analizados muestran en un $90.48 \%$ pequeñas frases de títulos o acciones a realizar en los cuadernos de actividades en letras mayúsculas, frente a un $81.08 \%$ que también lo muestran en minúsculas. Hemos podido observar, también, cómo el cambio de letra en algunos métodos se muestra de manera progresiva desde el primer curso hasta el tercero, evolucionando de la letra mayúscula, posteriormente a la letra minúscula y finalmente en algunos casos en cursiva.

En lo alusivo a las propuestas didácticas, observamos entre los materiales didácticos grandes diversidades, si bien, encontramos que en todos los materiales los objetivos son adecuados a la edad del alumnado, aunque, encontramos que para el desarrollo de una unidad o un proyecto que puede llevarse a cabo en quince días o un mes, se explicitan en una amplia lista de objetivos a conseguir o de contenidos a trabajar, que creemos que no se pueden dar como finalizados, sino como comenzados. También, encontramos que solo un $16.67 \%$ de los materiales muestran sus contenidos correlacionados entre sí, lo cual no facilita la globalización ni la generalización de los aprendizajes. Los maestros describen como más importantes, por parte de los materiales, a los contenidos procedimentales en esta etapa, superando a los contenidos conceptuales y actitudinales.

Es cierto que, tratando de evitar el reduccionismo material curricular = libro de texto, encontramos que, en Educación Infantil, los cuadernos de actividades (fichas) se encuentran adornados y rodeados de otros materiales muy variados, distribuidos en su uso para el alumnado, el profesorado o el aula.

Una de las principales novedades del currículo es la incorporación del enfoque de competencias en la Ley Orgánica de Educación (LOE, 2006), que, como hemos podido observar en las tablas de categorización, en Educación Infantil, muchos métodos las han adaptado junto a los objetivos como un apartado más en la programación.

Que tal como afirma Cardona (2016: 112), frente a quienes afirman la falta de una definición coherente del concepto (Mulder et al., 2008), la competencia (Tejada, 1999; Coll, 2007) integra el saber, saber hacer, saber estar y saber ser, y se define, en una primera aproximación al concepto, como un conjunto de conocimientos, procedimientos y actitudes que han de estar combinados, coordinados e integrados en el ejercicio profesional. 
La competencia podemos entenderla (De Miguel, 2005) como el conjunto de capacidades y de saberes técnicos, metodológicos, sociales y participativos que la persona pone en juego para afrontar las exigencias que le plantea el mundo laboral. Asimismo, ha gozado de consenso la clásica definición que aportó en su día la OIT (1998), al entenderla como aquella capacidad de integrar lo intelectual y lo emocional en términos de conocimientos, habilidades, actitudes y prácticas.

En lo referente a las actividades de aula, entendemos, de los datos obtenidos, que se debe dar una mayor profundidad a las actividades, sobre todo a aquellas referidas a generar interrogantes cognitivos, a la búsqueda de información, descontextualización y aplicación, síntesis y evaluación del alumnado, valorando cómo en el material se deberían incrementar así mismo las actividades de motivación de manera que no solamente sean iniciales.

Vemos muy positivamente tratados los materiales de lectura, habiendo aumentado en cantidad y calidad. Siendo muy favorable la valoración de los materiales informativos y resaltando su escasez, sería positivo que las editoriales siguieran apostando por ellos como material para mejorar la cientificidad de los aprendizajes del alumnado.

Dada la gran mezcla que se encuentra en los materiales respecto a la evaluación del alumnado, vemos necesario que se aporten mejores y más actividades de evaluación formativa y sumativa, y, sobre todo, consideramos que no se deben entremezclar, tal como encontramos en los materiales referentes de evaluación muy amplios con otros muy concretos.

En cuanto a los materiales complementarios, de carácter digital, hemos recogido cómo el $42.86 \%$ de los materiales del alumnado vienen acompañados de CD o DVD, mientras que el $79.60 \%$ de los materiales del profesorado vienen acompañados de material de programación y actividades para el alumnado, y el 69.39\% de los materiales del aula los anexan, siendo necesario que las Administraciones y los centros sigan promoviendo en estas edades el uso de dichos materiales digitales, por lo que no nos debemos quedar atrás en cuanto a dotaciones que permitan su uso en las aulas, ya que el profesorado en general se encuentra dispuesto y preparado para su uso.

Dada la situación de cambio social que se ha producido en los últimos tiempos y la pronta escolarización de los niños, valoramos como muy importante que los materiales curriculares se hagan eco de dichos cambios que se producen, ya que influyen de manera especial en la escuela. Como afirma Vila (2000: 14), la Educación Infantil es una respuesta intencionalmente educativa a las necesidades de cuidado de la primera infancia en el mundo moderno. Por ello consideramos que es necesario que los materiales curriculares se hagan eco de este cambio tratándose de adaptar a los diferentes niveles que los niños plantean al llegar al segundo ciclo de Educación Infantil; aunque en muchos casos se dispone ya de materiales en el primer ciclo de Infantil, vemos prioritario que los textos se pudieran adaptar a los diferentes ritmos de aprendizaje, ya que tal como apreciamos en los cuadros categoriales, solo el 25.64\% explicita distintos niveles para la realización de 
las actividades. Sería conveniente, a este respecto, hacer estudios en los que se valore en nuestra ciudad las diferencias entre el alumnado que ha asistido a alguna escuela infantil en el primer ciclo y los que no. Sí que existen estudios que miden la calidad en la Educación Infantil, tal como hace Lera (2007), basándose en instrumentos como la escala de evaluación de los entornos infantiles: Early Chilhood Environmental Rating Scale ECERS (Harms y Clifford, 1980) y la herramienta de Observación de Actividades Preescolares (OAP).

Indicar, también, que los aspectos más formales han sido valorados como adecuados en cuanto a su atractivo, adecuación del papel, letra, contraste texto o papel. Nos llama la atención que el encuadernado no sea en espiral y con portadas duras en todos los casos; tampoco aparece ninguna fotografía en blanco y negro en el texto.

En cuanto a la atención del alumnado que precisa de materiales de refuerzo y ampliación, hemos podido constatar que, en su mayor parte, disponen de dichos materiales, sobre todo en formato fotocopiable, mas nos planteamos si son del todo útiles, ya que no se adaptan a la individualidad de las necesidades tan específicas de estos alumnos. Se echan en falta en los textos medidas organizativas para abordar discapacidades motóricas, visuales o auditivas que se dejan en manos de los profesionales o de otro tipo de manuales, si bien, los materiales digitales y libros online facilitan estas adaptaciones por parte de los profesionales.

Una de las necesidades más primordiales que vemos que no se realiza con respecto a los materiales curriculares es escuchar a los propios alumnos, por ejemplo, en el paso a Educación Primaria. Basándonos en algunos datos del estudio de Argos et al. (2011) encontramos cómo las mayores inquietudes que muestran los niños ante el paso a Primaria son, entre otras: la preocupación por hacer amigos y por mantener los ya existentes, la creencia de que existirán normas que implicarán un mayor compromiso por su parte, así como el desarrollo de la lectoescritura, el conocimiento matemático, social y natural, siendo aspectos importantes que suponen un cambio en la metodología didáctica respecto a la Educación Infantil.

Finalmente, destacar la necesidad de dotar de mayor empaque científico a los materiales destinados a la Educación Infantil, ya que es el primer referente textual con que el niño va a trabajar en la escuela, y por ello no debe dar la sensación de que todo vale; muchas veces los textos lo tienen, mas no se hace evidente y creemos que se debería dar mayor importancia a aspectos como: la autoría, la bibliografía, la justificación del proyecto, basándose en aportaciones de diversos estudiosos, destacando de dónde proceden las imágenes, facilitando su uso y entendimiento a través de un índice o un diagrama de contenidos que ayude a ver en qué podemos ayudar al niño, destacando fichas de diversos colores, tamaños o formas, ya que la igualdad no fomenta la autonomía de uso por parte del alumnado. En cualquier caso, los materiales curriculares deberán adaptarse a los nuevos tiempos, y por supuesto a las características, intereses y necesidades del niño, que es el principal protagonista del proceso de enseñanza-aprendizaje. 
Por último, tras el estudio realizado, caben destacar algunas reflexiones del profesorado de Educación Infantil ante la elección de un determinado material curricular, resumiéndose en las siguientes:

1. Me permite evitar el reduccionismo material curricular $=$ libro de texto.

2. Establece variadas unidades didácticas, proyectos, etc., que se adecúan a mi realidad educativa.

3. Me permite alcanzar los objetivos y competencias que quiero conseguir en la etapa, de acuerdo al alumnado y el contexto.

4. Es adecuado para transmitir los contenidos que me propongo y tienen valor educativo.

5. Me aporta una visión desde las competencias básicas, inteligencias múltiples, ejes transversales u otras visiones que yo tengo en cuenta para mi programación.

6. Los centros de interés que se proponen son variados y actualizados.

7. Las actividades tienen profundidad, adecuándose a los diferentes momentos: motivación, evaluación inicial, memorización, búsqueda de información, plantean interrogantes cognitivos, de contextualización y aplicación, de síntesis, de evaluación formativa, etc.

8. La cantidad y calidad de material científico y de lectura que me aporta es suficiente y adecuada a las características textuales que necesito.

9. El material de apoyo (refuerzo, ampliación e individualización) es variado y apropiado para que el alumnado pueda tener diferentes ritmos de aprendizaje y niveles de ejecución.

10. Los materiales anexos me ayudan a que el alumnado consiga más fácilmente las competencias y los objetivos propuestos.

11. La evaluación que se propone en el material del proceso educativo se adapta a mis necesidades de contexto en el centro y en mi aula.

12. Tiene los aspectos formales que necesito (encuadernación, tamaño, color, etc.)

13. Fomenta la autonomía de uso para el alumnado.

14. Contiene el empaque científico que considero suficiente (bibliografía, justificación del proyecto, referencia a algún estudio, etc.).

15. Me permite coordinarme con mis compañeros de ciclo y etapa de Educación Primaria.

\section{REFERENCIAS BIBLIOGRÁFICAS}

Antón Sancho, A. y Gómez Alonso, M. (2016). La geometría a través del arte en Educación Infantil. Enseñanza \& Teaching, 34, 1-2016, 93-117.

Argos González, J.; Ezquerra Muñoz, M. P. y Castro Zubizarreta, A. (2011). Escuchando la voz de la infancia en los procesos de cambio e investigación educativos. Aproximación al estudio de las transiciones entre las etapas de Educación Infantil y Educación 
TERESA ALONSO PEDROSA Y ROSA M. ${ }^{a}$ SANTAMARÍA CONDE

Primaria. Revista Iberoamericana de Educación, 54 (5). Descargado el 5 de diciembre de 2016. http://rieoei.org/index.php.

Ballesta Pagán, J. (1995). Función didáctica de los materiales curriculares. Pixel-Bit. Revista de Medios y Educación, 5, 29-46.

Bardin, L. (1986). Análisis de Contenido. Madrid: Akal.

Boronat Mundina, J. (2005). Análisis de contenido. Posibilidades de aplicación en la investigación educativa. Revista Interuniversitaria de Formación del Profesorado, 19 (2), 157-174.

Cabero Almenara, J. y Loscertales Abril, F. (2002). Elaboración de un sistema categorial de análisis de contenido para analizar la imagen del profesor y la enseñanza en la prensa. En La investigación en medios de comunicación. Descargado el 14 de abril de 2017. http://tecnologiaedu.us.es/cuestionario/bibliovir/57.pdf.

Cardona Andújar, J. (2016). Formación en competencias comunicativas de los maestros de Educación Infantil. Enseñanza \& Teaching, 34, 2-2016, 109-130.

Delgado de Paiva, M. y Rodríguez Rodríguez, J. (2011). La consideración de las dificultades de aprendizaje en una muestra de libros de texto y materiales didácticos de educación primaria en Portugal. Enseñanza \& Teaching, 29, 2-2011, 103-123.

Gútiez Cuevas, P. (1995). La Educación Infantil: modelos de atención a la infancia. Revista Complutense de Educación Infantil, 6 (1), 101-113.

Jaramillo, L. (2007). Concepción de infancia. Zona próxima. Revista del Instituto de Estudios Superiores en Educación. Universidad del Norte, 8, 108-123.

Krippendorff, K. (1990). Metodología de análisis de contenido: teoría y práctica. Barcelona: Ediciones Paidós.

Lera Rodríguez, M. J. (2007). Calidad de la Educación Infantil: Instrumentos de evaluación. Revista de Educación, 343, 301-323.

Ley Orgánica 1/1990, de 3 de octubre, de Ordenación General del Sistema Educativo ( $B O E$ n. ${ }^{\circ} 238$, de 4 de octubre).

Ley Orgánica 2/2006, de 3 de mayo, de Educación ( $B O E$ n. ${ }^{\circ}$ 106, de 4 de mayo).

Ley Orgánica 8/2013, de 9 de diciembre, para la Mejora de la Calidad Educativa (BOE n. ${ }^{\circ}$ 295 , de 10 de diciembre).

Martínez Bonafé, J. (1992). Siete cuestiones y una propuesta. Cuadernos de Pedagogía, 203, 8-13.

Martínez Bonafé, J. (2001). Óxido sobre el currículum en plena era digital (Crítica a la tecnología del libro de texto). Kikiriki. Cooperación Educativa, 61, 22-29.

Martínez Redondo, M.; Pérez Ferra, M. y Sierra y Arizmendiarrieta, B. (2014). Incidencia de los hábitos educativos en el aprendizaje del alumnado de Educación Infantil. Ensenanza \& Teaching, 32, 2-2014, 147-176.

Parcerisa Aran, A. (1999). Materiales curriculares. Cómo elaborarlos, seleccionarlos y usarlos. Barcelona: Editorial Graó.

Salgado Somoza, M. y Salinas Portugal, M. J. (2009). El número en los libros de texto de Educación Infantil. En M. J. González, M. T. González y J. Murillo (Coords.). Investigación en Educación Matemática (tomo XIII, pp. 487-497). Santander: SEIEM.

Vila, I. (2000). Aproximación a la educación infantil: características e implicaciones educativas. Revista Iberoamericana de Educación, 22. Descargado el 18 de diciembre de 2016. http://rieoei.org/rie22f.htm. 